\title{
LV state estimation and TSO-DSO cooperation tools: results of the French field tests in the evolvDSO project
}

\author{
Maria Viania Sebastian ${ }^{\boxplus}$, Mathieu Caujolle ${ }^{2}$, Belén Goncer Maraver ${ }^{2}$, \\ Jorge Pereira ${ }^{3}$, Jean Sumaili4 ${ }^{4}$ Pedro Barbeiro ${ }^{4}$, João Silva ${ }^{3}$, \\ Ricardo Bessa 4 \\ ${ }^{1}$ Enedis, Paris, France \\ ${ }^{2}$ EDF Lab, Saclay, France \\ ${ }^{3}$ INESC TEC and FEP, Porto, Portugal \\ ${ }^{4}$ Inesc TEC, Porto, Portugal \\ 凶-mail: maria.sebastian-viana@enedis.fr
}

\begin{abstract}
This study presents the results of field tests performed on French medium-voltage distribution networks with two novel algorithms developed in the framework of the evolvDSO Project. Working in the transmission system operator and distribution system operator (TSO-DSO) cooperation domain, the interval constrained power flow tool estimates the flexibility range at primary substations by aggregating the distribution network flexibility. The lowvoltage state estimator tool evaluates the voltage profile of a low-voltage (LV) network using an artificial neural network trained on historical data. Based on real-field data and considering various real-life scenarios, both algorithms look promising in terms of efficiency and scalability. Areas of improvement were also identified.
\end{abstract}

\section{Introduction}

Distribution system operators (DSOs) face new challenges with the increase of distributed generation (DG), the usage of new equipment such as electrical vehicles (EVs), the emergence of storage systems as well as new market players such as flexibility aggregators. However, thanks to Smart Electric Grids, the DSOs will have additional capabilities to monitor and control the distribution grid at both medium-voltage (MV) and low-voltage (LV) levels by relying on advanced information and communication technology architectures, and innovative algorithms.

Within the framework of the FP7 EU Project evolvDSO (www. evolvdso.eu), Enedis experimented on field data two original algorithms developed by INESC TEC. The first one, called interval constrained power flow (ICPF), belongs to the transmission system operator and distribution system operator '(TSO-DSO) cooperation' domain [1]: it aggregates the distribution network flexibility and estimates a region of feasible values for the active and reactive powers exchanged at the boundary nodes between the transmission and distribution networks, as well as its associated costs. The second one, the low-voltage state estimator (LVSE), aims at improving the real-time observability of LV grids [2]. It evaluates their voltage profile, even if they are poorly characterised.

This paper provides the results collected by applying both algorithms to field data: one LV site consisting of several LV substations was considered for the LVSE, and two MV networks, each including at least one primary substation and considering different flexibility asset types, were used for the ICPF.

\section{Interval constrained power flow}

\subsection{Algorithm description}

The ICPF tool aims to increase the cooperation between TSOs and DSOs by favouring their interaction at different timeframes, thus improving the system security. It estimates the active and reactive power flexibility ranges available at each primary substation, i.e. at the TSO-DSO interface, and the main associated cost areas.

This process is performed for several hours ahead in operational planning by considering: (i) the planned network topology, (ii) the local consumption and generation forecasts, (iii) the technical grid and asset constraints, (iv) the flexible resources available in each downstream distribution network and (v) their associated costs. The flexibility levers the tool is able to consider are divided into three types: the usual technical DSO levers [on-load tap changers (OLTCs) and capacitor banks], the regulated ones (e.g. firm and non-firm connection contracts) and the market based ones. It then performs a technical and economic evaluation of the flexibility from the bulk power system point of view. More information about the ICPF tool features can be found in [3].

\subsection{Field test settings}

Various tests were carried out on two MV networks in order to assess the performance achieved by the tool: (i) a small rural network, and (ii) a large urban network. Their characteristics are provided in Table 1.

Several flexibility assets were considered within the framework of the trials:

- DSO owned assets: OLTCs of the high-voltage (HV)/MV transformers and reactive power compensation (e.g. MV capacitor banks);

- Distributed $M V$ generators: active and reactive power flexibility; - Battery storage system (only on rural network): active power flexibility of the battery charge/discharge. The reactive power flexibility is not considered for this asset in the tool.

It is important to note that the usage of some of these levers (in particular, those provided by third parties) is currently not possible for DSOs in the context of the French regulatory framework. Here, these levers are only considered in a prospective way. 
Table 1 Main characteristics of studied networks

\begin{tabular}{lll}
\hline & Simple MV & Large MV \\
\hline primary substations & 1 & 2 \\
HV/MV transformers & 1 (20 MVA) & 5 (36 MVA each) \\
MV feeders & 6 & 5 \\
MV/LV substations & 114 (total of 6.5 MVA) & 577 (total of 62 MVA) \\
MV customers & 15 (total 3.5 MVA) & 106 (total 34 MVA) \\
MV production units & 1 wind farm 12 MW & 1 cogeneration 1 MW \\
& 1 wind farm 6 MW & $1 \mathrm{PV} 1.3 \mathrm{MW}$ \\
capacitor banks & $1(2.4 \mathrm{MVAr})$ & $1 \mathrm{PV} 4.2 \mathrm{MW}$ \\
battery storage & $1(1.3 \mathrm{MWh})$ & 2 units of $1.8 \mathrm{MVAr}$ \\
& & 3 units of 4.8 MVAr \\
\hline
\end{tabular}

Many scenarios were considered involving various combinations of production levels, asset availability, associated costs and network topologies. All were performed a posteriori using the following field data:

- Current topology of the network fetched from Enedis SCADA system, completing the static description extracted from Enedis GIS;

- Up-to-date load and generation forecasts computed by Enedis forecasting system;

- Parameters associated to each flexibility asset (variation domain and associated costs)

\subsection{Field test evaluation}

Two performance metrics (PMs) were defined to assess the ICPF effectiveness:

- Computational time required to provide the flexibility cost map for a specific time instant;

- Size of the estimated flexibility area.

Both PMs are compared to those obtained with a classical Monte-Carlo approach (considering from 1000 to 100,000 samples) to quantify the gain. It will help us to assess, respectively, the scalability of the tool, and the accuracy of the results it provides.

\subsection{Field test results}

The tool performed well for the different test cases considered, replicating real-life situations of the studied MV networks. Flexibility cost maps such as the one displayed in Fig. 1 were provided by the tool for each hour of the predefined time horizon: in addition to the reference operating point, four flexibility areas

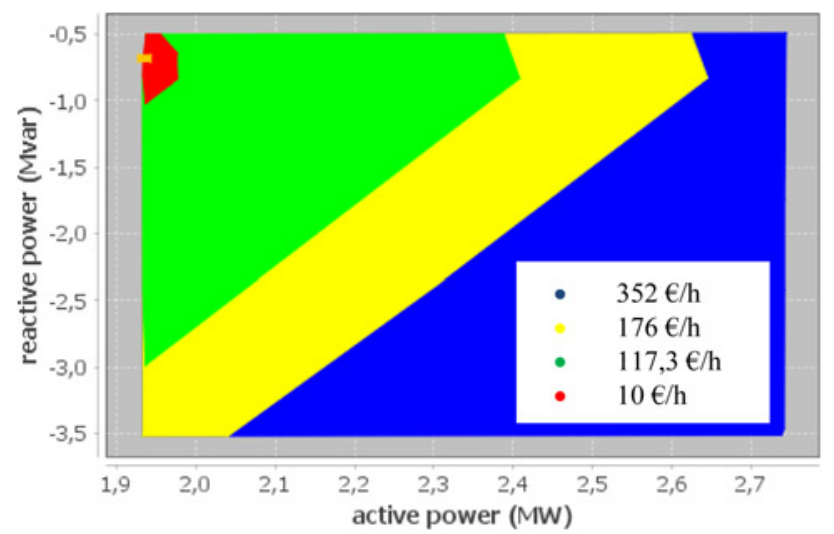

Fig. 1 Example of flexibility cost map obtained for a particular configuration of the rural MV network with DSO assets and storage flexibility considered
Table 2 PMs obtained for different scenarios applied to the rural MV network

\begin{tabular}{|c|c|c|c|c|c|c|}
\hline \multirow{2}{*}{$\begin{array}{l}\text { Scenario } \\
\text { samples nb }\end{array}$} & \multicolumn{3}{|c|}{ Flexibility area increase, \% } & \multicolumn{3}{|c|}{$\begin{array}{l}\text { Computational time } \\
\text { reduction, } \%\end{array}$} \\
\hline & $1 \mathrm{k}$ & $10 \mathrm{k}$ & $100 \mathrm{k}$ & $1 \mathrm{k}$ & $10 \mathrm{k}$ & $100 \mathrm{k}$ \\
\hline DSOFlex & 24.68 & 5.59 & 2.86 & 88.88 & 98.97 & 99.9 \\
\hline DSO\&GenFlex & 319.84 & 197.12 & 162.13 & 91.57 & 99.1 & 99.9 \\
\hline DSO\&BatFlex & 32.08 & 14.37 & 10.07 & 90.36 & 98.98 & 99.9 \\
\hline AllFlex & 117.79 & 51.94 & 32.98 & 92.03 & 99.21 & 99.92 \\
\hline
\end{tabular}

are provided, each delimited by a maximum price. Three of these prices are automatically calculated by the ICPF tool. They correspond to the maximum price achievable when activating all the flexibility assets, and to the half and the third parts of this price. The fourth price is defined by the control centre operator. It can be larger, smaller or in-between any of these three prices. Therefore, the largest of these four areas corresponds to the maximum flexibility domain achievable for this network for the topology planned at this hour.

(i) Lessons learned on the flexibility map accuracy: The flexibility areas provided by the ICPF are the largest even when compared to the Monte Carlo approach (described in [4]) using the largest sample number (Table 2). This increase is the most significant when the maximum number of flexible assets is considered (AllFlex scenario), showing the added value of the ICPF.

Only one minor issue was encountered regarding the DER units flexibility when both active and reactive powers are considered as flexible: the rated power of the unit might be overcome in some situations. Some additional parameters should be introduced in order to better tune the power electronics and the generation part of these units.

(ii) Lessons learned on the computational requirements: The ICPF approach is quite efficient in comparison to MC: it needs less time to compute a more accurate flexibility map (Table 2).

The flexibility map computational time mainly depends on two factors: the network size and the number and type of the flexible assets considered. With a simplified version of the networks under study, hourly flexibility maps computations require from 5 to 30 min for each substation on a standard PC. 'Simplified version' means that the non-controllable switches are removed from the network representation and the line series are merged to reduce at maximum the number of network assets. Such operation has thus to be performed for each network topology. With the full network description, the computation time almost doubles: it goes up to $55 \mathrm{~min}$ for the largest substation.

If such computational times are reasonable for hourly forecasts (all the more since parallel computation of several maps is possible with the tool), it might become problematic if shorter time steps (30 min, or even $15 \mathrm{~min}$ ) were considered. Simplifying the network beforehand would thus become a requirement.

\section{Low-voltage state estimation}

\subsection{Algorithm description}

The LVSE tool developed in the context of the evolvDSO project evaluates the voltage profile of a complete LV network. It follows an innovative approach based on an artificial neural network trained on historical data [1,2]. It uses limited information when compared to traditional state estimation techniques, such as weighted least squares algorithms:

- It requires no detailed electrotechnical or topology data of the network, only the connection phase of the customers, and 
historical voltage and power data collected by the smart meters (SMs);

- It exploits the voltage and power quantities collected in real time by SMs.

The first stage of the LVSE algorithm consists in training the neural network based on the historic database (Fig. 2). This database needs to contain sufficient synchronised data about the variables (voltage and powers) that will be passed to the model during its training stage, including those considered in real time.

The training relies on the use of AutoEncoders (AE) trained through extreme learning machine (ELM) techniques. This off-line process allows to learn the patterns/dependencies between the electrical variables of a given network, and performs a reliable state estimation.

In the second stage, the trained $\mathrm{AE}$ is used as the 'brain' of the LVSE algorithm. The measurements provided in real time by SMs are used as its inputs to compute the state estimation solution. The solution comprises the state variables to be estimated: voltage magnitude values, as well as the active power and reactive power injections if desired.

\subsection{Field test settings}

The site considered for the trials of the LVSE tool consisted of 11 MV/LV substations. This represents about 1250 LV customers, most being residential and the rest being of the commercial type. They are split between about 50 feeders and $15 \mathrm{~km}$ of LV lines. No photovoltaic generation, storage or EV unit is present on these networks. About 1000 of the customers were equipped with SMs allowing their phase connection to be known. These information are not available for the customers not equipped with a SM.

To allow extensive testing of the LVSE, reference datasets were constituted for these networks by performing load-flow calculation based on real data. In addition to their description, we used the following data collected from the field:

- Power measurements with a 30 min time step;

- Three-phase LV voltage measurements collected at LV substation bus bars with a $10 \mathrm{~min}$ time step.

This allowed us to simulate network behaviour as near to real life as possible and to investigate a large number of strategies regarding: the number and positioning of real-time measurements (RTMs), the number and positioning of SMs considered for the training, the

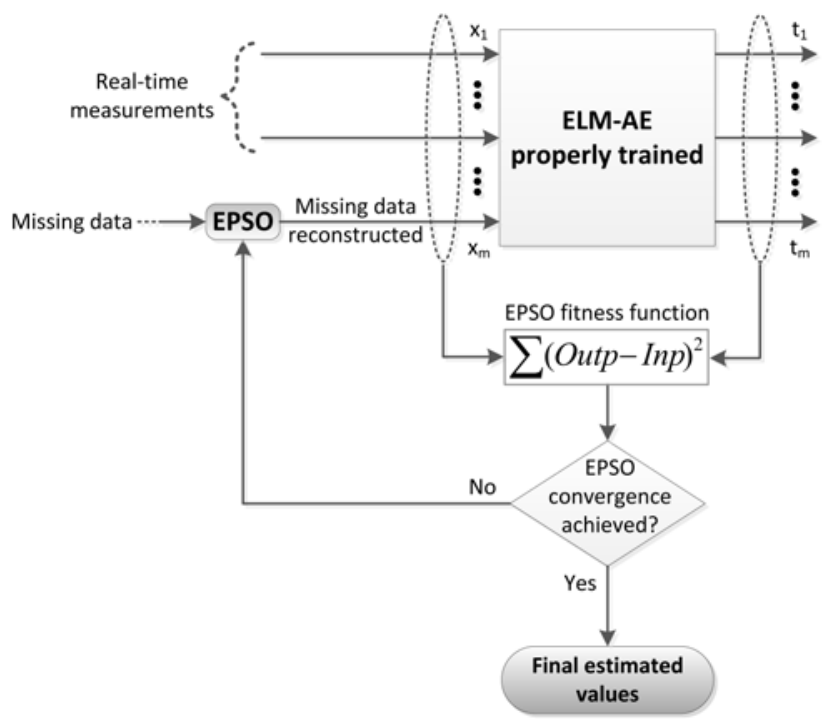

Fig. 2 ELM-AE methodology illustration historic database duration and so on This extensive testing could not have been done directly on the field given the experimental constraints and their limited timeframe.

\subsection{Field test evaluation}

A consistent database of over 18 months was constituted to evaluate the algorithm. Out of these 18 months, the first year was used as reference to test different training strategies. The next 6 months were used to consistently assess the state estimations provided by the tool in the different configurations.

Several PMs were considered to assess LVSE efficiency:

- 'Accuracy' PMs to characterise the estimation error on the voltage magnitude;

o Min/max estimation error (V),

o Average estimation error (V),

o Standard deviation of the estimation error (V),

- Distribution of the estimation error,

- 'Computational' PMs to characterise the LVSE computation process;

○ Average pre-processing duration (s),

o Average training duration (s),

- Average and standard deviation of the state estimation duration (s).

\subsection{Field test results}

(i) Lessons learned on training strategies: Trials of the LVSE have highlighted that both the estimation accuracy and the training process duration substantially improve when independent computations of the network feeders are performed instead of considering the whole network.

Similar improvements were observed when limiting the redundancy of information during the training, i.e. when selecting only one SM per node and per phase while ensuring maximum observability of the network (Fig. 3).

The analysis of the training strategies proved that the ELM-AE model requires only a short amount of data to provide a reliable estimation: two days data seem already sufficient so that the LVSE can provide again reliable state estimation over the next month (Fig. 4). This is a strong requirement since the LVSE neural network model should be adapted in case the LV network topology evolves, i.e. a new customer or a piece of new equipment such as EVs is added.

(ii) Lessons learned on real-time observation strategies: Only a reduced number of RTMs is required to provide sufficient accuracy. Three RTMs (one on each phase of a feeder) already provide exploitable results. Nine RTMs (three measurements on each phase dispersed on the feeder) were sufficient to provide accurate estimation on a feeder of 150 customers (Fig. 5).

The location of the RTMs seems to have a limited impact on the state estimation accuracy. If located in the middle of the feeder or at its end, the overall accuracy is quite similar.

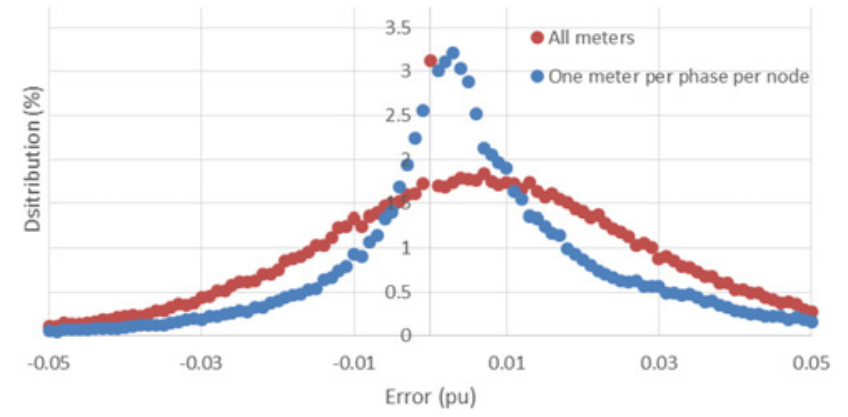

Fig. 3 Impact of SM redundancy on the state estimation accuracy 


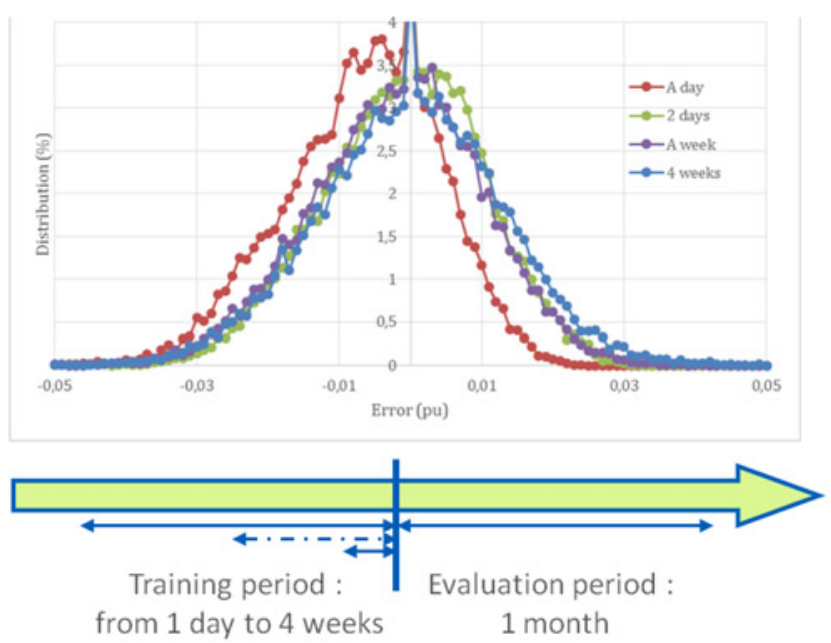

Fig. 4 Impact of training periods on the estimation accuracy for an evaluation period of 1 month

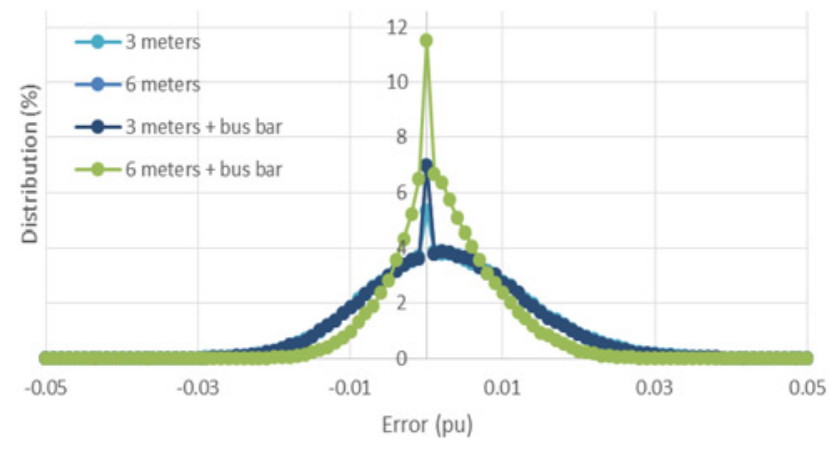

Fig. 5 Impact of the number of SMs transmitting data in real time on the state estimation accuracy

(iii) Lessons learned on the computational requirements: The pre-processing stage of the database is independent from the LVSE algorithm itself, but it plays a key role. It ensures that the time interval considered for the ELM-AE training is the intersection of the time intervals where measurement data is present for all the selected SMs. This current requirement makes it quite time consuming: the larger the number of SMs considered for the training, the longer the training data consolidation will last (Fig. 6a). This operation should be optimised in case of an operational deployment.

Once the database has been consolidated, the training process itself is quite efficient: it lasts $<1 \mathrm{~min}$ for a database consisting of about 300 measurement locations and 1 year of data. Its duration evolves nearly linearly with the number of considered SMs (Fig. 6b).

Such an evolution pattern could not be found for the LVSE RT state estimation computation (Fig. 6c). This process is quite fast: it lasts at most $12 \mathrm{~s}$ for a network of 300 customers. Over the numerous situations investigated during the tests, a computation time of $1.4 \mathrm{~s}$ was observed in average.

\section{Conclusions}

The ICPF tests showed that the tool fulfils its expectations by providing the aggregated active and reactive power flexibility available at the TSO-DSO boundary and its associated flexibility cost map in a reasonable amount of time. The calculation of the flexibility map for a primary substation lasts no longer than 30 min, making it compatible with the time constraint of operational planning.

The ICPF tool could be enhanced in several ways, such as: (i) speed-up the flexibility map calculation; (ii) manage battery state of charge schedules and use storage reactive power as a flexibility lever; (iii) automate the integration of scheduled network reconfigurations, for example happening because of maintenance; (iv) manage the generation unit flexibility so that their rated power is never exceeded; (v) make the GUI more user-friendly, e.g. allow setting the cost ratios of the flexibility areas. INESC TEC has already started to work on the speed-up by developing a new sparse solver for optimal power flow (OPF) calculations.

Based on the feedback of LVSE tests, the accuracy, the robustness to RTM losses and the computational requirements (processing power, memory requirement etc.) of the algorithm seem promising with regard to a decentralised application in equipment at the MV/LV substation level. More testing, involving networks with DG and active loads, would however be required for a definite answer.

Areas of improvements were also identified during the tests, the main concerning the LVSE training. The current constraints on data presence and synchronicity would make it difficult to apply on the field where communication issues, missing data and invalid data are likely to be encountered. Different solutions are being investigated by INESC TEC (improved usage of customer location/connection etc.) to limit this constraint.

\section{Acknowledgments}

The research leading to this work has been carried out as a part of the evolvDSO project (Development of methodologies and tools for new and evolving DSO roles for efficient DRES integration in distribution networks) - www.evolvdso.eu. This project is funded by the European Commission under the
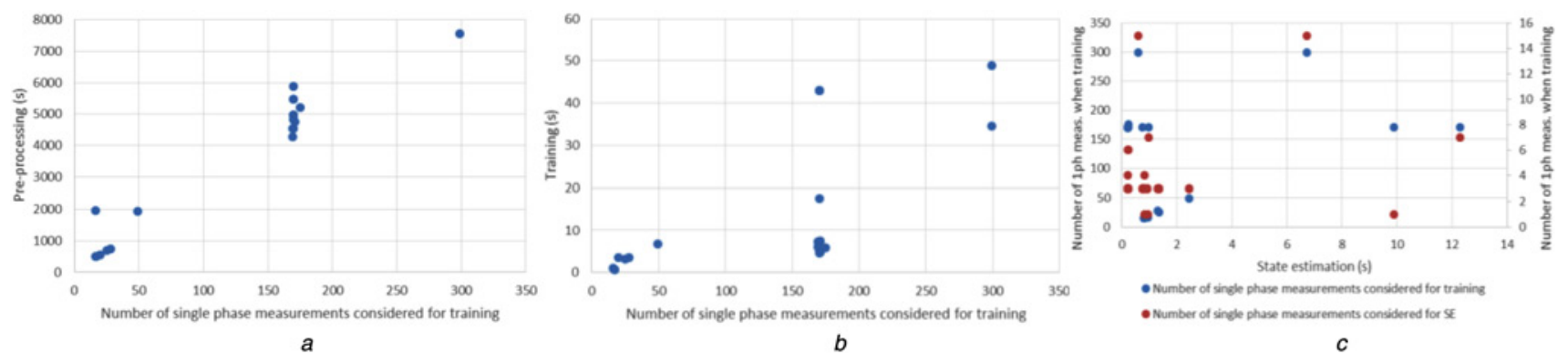

Fig. 6 Dependency on the measurement number in a 300 customers' network regarding:

(a) Pre-processing duration, (b) Training duration, (c) State estimation duration 
seventh framework program (FP7) under grant agreement number 608732 .

\section{References}

1 Barbeiro, P.N.P., Teixeira, H., Pereira, J., et al.: 'An ELM-AE state estimator for real-time monitoring in poorly characterized distribution networks'. Proc. IEEE Eindhoven PowerTech, 2015, pp. 1-6
2 Teixeira, H., Barbeiro, P.N.P., Pereira, J., et al.: "Advanced tools and methodologies for forecasting, operational scheduling and grid optimisation', Deliverable D3.2 of the evolvDSO Project, 2015

3 Sumaili, J., Silva, J., Bessa, R., et al.: 'Advanced methodologies and tools for operation and maintenance of distribution grids with DRES'. Deliverable D3.3., FP7 EU Project evolvDSO, September 2015

4 Heleno, M.D., Soares, R., Sumaili, J., et al.: 'Estimation of the flexibility range in the transmission-distribution boundary'. Proc. IEEE PowerTech 2015, Eindhoven, Netherlands, June 2015. 